\title{
Directional coherent light via intensity-induced sideband emission
}

\author{
Andrew J Traverso ${ }^{1}$, Chris O’Brien ${ }^{1}$, Brett H Hokr ${ }^{1}$, Jonathan V Thompson ${ }^{1}$, Luqi Yuan ${ }^{1}$, Charles W Ballmann ${ }^{1}$, \\ Anatoly A Svidzinsky ${ }^{1}$, Georgi I Petrov ${ }^{1}$, Marlan O Scully ${ }^{1,2,3}$ and Vladislav V Yakovlev ${ }^{1}$
}

We introduce a unique technique for generating directional coherent emissions that could be utilized to create coherent sources in a wide range of frequencies from the extreme ultraviolet (XUV) to the deep infrared. This is accomplished without population inversion by pumping a two-level system with a far-detuned strong optical field that induces the splitting of the two-level system. A nonlinear process of four-wave mixing then occurs across the split system, driving coherent emission at sidebands both redand blue-detuned from the pump frequency, and propagates both forward and backward along the pump beam path. We observed this phenomenon in dense rubidium vapor along both the $D_{1}$ and $D_{2}$ transitions. The sideband emission exhibits a short pulse duration $(<1 \mathrm{~ns})$ with threshold-like behavior dependent on both the pump intensity and $\mathrm{Rb}$ vapor density. This technique offers a new capability for manipulating the emission frequency simply through intensity-induced atomic modulation that can be scaled to most frequency regimes using various atomic/molecular ensembles and pump energies. Light: Science \& Applications (2017) 6, e16262; doi:10.1038/Isa.2016.262; published online 19 May 2017

Keywords: coherent emission; collective coherence; four-wave mixing

\section{INTRODUCTION}

New approaches for generating coherent light are of keen interest to the scientific community due to the ever-increasing demand for unique sources tailored to specific applications. In certain fields, such as remote sensing and soft X-ray/extreme ultraviolet (XUV) lasers, this interest is especially pronounced as a novel technique would have a profound impact ${ }^{1-4}$. Here, we describe a unique method for generating directional coherent emissions that could be utilized for these and other applications. This approach offers two distinct advantages: it does not require population inversion, and the coherent light is produced simply by optically pumping a two-level transition with a strong field that is far-detuned from resonance. Once produced, the light propagates in both the forward and backward directions along the pump beam and is shifted in frequency further away from resonance compared with the pump field, that is, if the pump is blue- (red-) detuned from the transition, the emission is further blue(red-) detuned. We demonstrate detunings of up to $30 \mathrm{~nm}$ with a 5$\mu \mathrm{J}$, 6-ps pump in rubidium ( $\mathrm{Rb}$ ) vapor. Larger detunings and different wavelength regimes are achievable by scaling up the pump energy and employing different atomic/molecular ensembles.

The main difficulty in realizing a tabletop X-ray laser by conventional methods is achieving population inversion due to the extremely short lifetimes of the XUV/X-ray transitions. As such, there have been many proposed alternative methods ${ }^{5-7}$ that attempt to overcome this constraint, including plasma-assisted lasing ${ }^{8-10}$, which achieves population inversion by ionization/recombination, and high-harmonic generation $^{11,12}$, which bypasses the need for population inversion altogether. Despite these demonstrations, there is still a limited selection of accessible wavelengths and an overall lack of sources within this regime.

The use of advanced nonlinear techniques in atmospheric remote sensing has similarly been hindered by the lack of a practical method to generate a counter-propagating coherent source. Whereas there has been some marked success in developing such a technique utilizing the oxygen and nitrogen in the atmosphere as a gain medium ${ }^{13-16}$, these techniques rely on an intense ultraviolet (UV) pump source, which is not feasible due to the high absorption and scattering rates in the atmosphere ${ }^{17}$. A technique that produces a counter-propagating source without the need for a UV pump would have a substantially easier path to practical implementation.

In this study, we propose and demonstrate a unique approach for coherent light generation achieved by coupling a far-detuned intense pump field to a two-level system in the transient regime. The pump field induces AC Stark splitting in the system and subsequently drives the system through a four-wave mixing (FWM) process across the transiently split levels. The medium then emits directional, coherent light detuned from the pump frequency by the effective Rabi frequency on a timescale faster than the decoherence time of the system, hence the moniker fast Rabi-induced sideband emission (FRISBE). FRISBE is bidirectional in nature, does not require population inversion and should be attainable in most atomicl molecular systems. Given these attributes, it is a prime candidate for

${ }^{1}$ Texas A\&M University, College Station, TX 77843, USA; ${ }^{2}$ Princeton University, Princeton, NJ 08544, USA and ${ }^{3}$ Baylor University, Waco, TX 76798 , USA

Correspondence: V Yakovlev, Email: yakovlev@tamu.edu

Received 25 July 2016; revised 4 November 2016; accepted 30 November 2016; accepted article preview online 5 December 2016 
remote sensing and XUV/X-ray applications, as well as a potential alternative to other technologies such as parametric generation and chirped pulse amplification.

It has been demonstrated that it is possible to achieve a gain from a strongly driven two-level atom in the absence of population inversion. This effect is called 'Mollow gain' and was first shown in sodium gas $^{18,19}$. Mollow gain is essentially a single-atom process, but it was predicted $^{20,21}$ that for propagation through dense media it can be a source of gain that drives nonlinear FWM, leading to sideband generation. Mollow-driven FWM was originally proposed as a way to explain the 'anomalous' sideband generation observed in atomic vapor; however, it is still currently unclear whether this was the primary mechanism, as several plausible alternatives were also suggested $^{22-25}$. The difficulty in identifying the primary mechanism is because most of the experiments were performed with pump detunings of less than $300 \mathrm{GHz}$, where other significant effects come into play such as self-focusing, self-trapping and substantial changes to the refractive index. Consequently, the observed sideband generation was always near resonance and often led to conical emission for one of the sidebands ${ }^{26,27}$. In contrast, our studies focus on pump detunings greater than $300 \mathrm{GHz}$, where near-resonant effects have a negligible role. The resulting sideband emission is observed only along the beam path and with spectral detunings two orders of magnitude greater than those in previous experiments. To adequately explain our observations, we extend the approach in Refs. 20,21 into the nonsteady-state regime and to the case of large pump detunings.

\section{MATERIALS AND METHODS}

To understand this phenomenon, it is useful to consider a two-level system with a transition frequency $\omega_{a b}$ between the ground state $|b\rangle$ and excited state $|a\rangle$ driven by a strong field $\nu_{\mathrm{d}}$ (Figure 1a). If the field is strong enough such that the Rabi frequency, $\Omega_{\mathrm{d}}=\frac{\wp \cdot E}{\hbar}$, is comparable to detuning, $\Delta=\left|\omega_{a b}-\nu_{\mathrm{d}}\right|$, then the field will induce AC Stark splitting in both the ground and excited states ( $E$ is the electric field amplitude and $\wp$ is the electric dipole moment of the transition). Given the energy-level splittings, it is convenient to use an informal 'dressed state' picture for a better qualitative illustration of the dynamics (Figure 1a). In the dressed state description, the bare twolevel system transforms into a four-level system, where the levels are defined by the eigenstates of the convolution of the atomic system with the driving field. This gives a level splitting equal to the effective Rabi frequency, $\Omega_{\mathrm{Eff}}=\sqrt{\Omega_{\mathrm{d}}^{2}+\Delta^{2}}$, for each of the doublets.

Conceptually, this process is analogous to a coherent Raman process driven at resonance, where the pump acts as both the pump and probe fields ${ }^{20,27}$. The significant difference is that the energy levels in the dressed state picture only exist during the lifetime of the coherence. Initially, the strong drive field induces Mollow gain in the

a
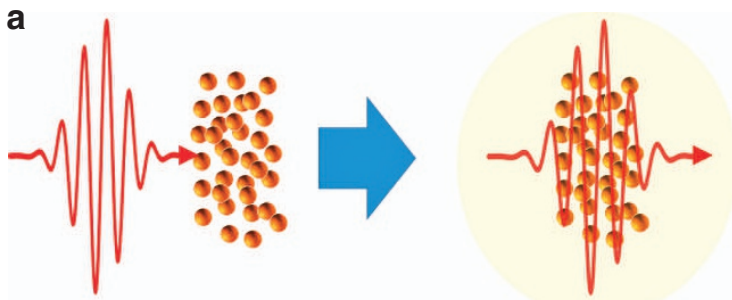

Two-level state Dressed state

Two-level state

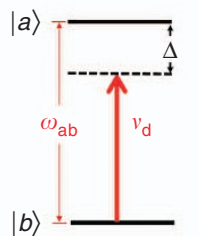

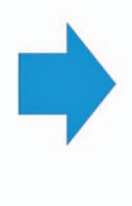

$|a\rangle$

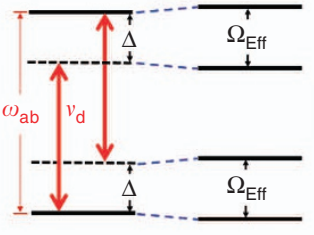

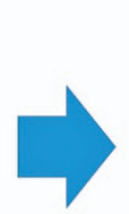

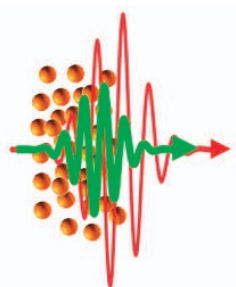

Four-wave mixing

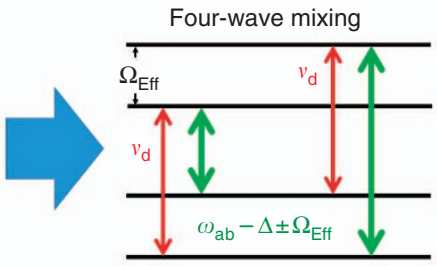

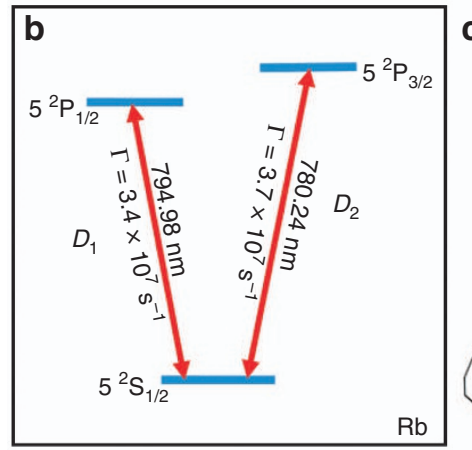
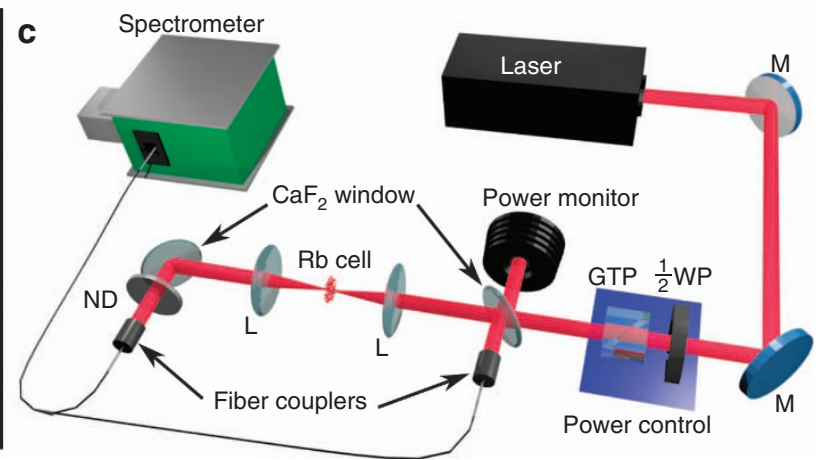

Power control

Figure 1 Experimental scheme. (a) Evolution of a two-level system into a split four-level picture where both the upper and lower doublets are split by the effective Rabi frequency $\Omega_{\mathrm{Eff}}$. Resonant four-wave mixing then occurs, where the pump laser acts as both the pump and probe field. (b) Simplified energylevel diagram of the rubidium atom. Pump laser is tuned from below the $D_{1}$ line to above $D_{2}$ line. (c) Diagram of experimental set-up. A tunable CW ECDL near $780 \mathrm{~nm}$ is used to seed an OPA comprising of a LBO crystal pumped with a $1 \mathrm{~mJ} 6 \mathrm{ps} 532 \mathrm{~nm}$ laser. ECDL, extended cavity diode laser; GTP, Glan-Thompson polarizer; L, lens; LBO, lithium triborate; M, mirror; ND, neutral density filter; $\frac{1}{2}$ WP, half-wave plate. 
medium, producing a weak omni-directional field at the effective Rabi frequencies. Then, due to the interaction between the dressed-state energy levels and the strong pump field, the Rabi-detuned fields are amplified by FWM in the transient regime while propagating along the pencil-like pump region. The resulting emission is both in the forward and backward directions, with frequencies expressed as $\nu_{\mathrm{d}} \pm \Omega_{\mathrm{Eff}}$, whereas absorption in the gain region occurs at $\nu_{\mathrm{d}}$. For this process to transpire, the coherence must play a significant role, meaning that both the Rabi frequency, $\Omega_{\mathrm{d}}$, and the collective atomic drive frequency, $\Omega_{\mathrm{A}}$ (defined later), must be much greater than the largest dephasing rate in the system 28,29 .

To demonstrate FRISBE generation, we chose to study the process in $\mathrm{Rb}$ vapor, as it has a high vapor density at relatively low temperatures and possesses two strong near-infrared transitions from the ground state (see Figure 1b): $D_{2}\left(5^{2} S_{1 / 2} \rightarrow 5^{2} P_{3 / 2}\right)$ and $D_{1}\left(5^{2} S_{1 / 2} \rightarrow 5^{2} P_{1 / 2}\right)$. Given that the $5^{2} P_{1 / 2} \rightarrow 5^{2} P_{3 / 2}$ transition in $\mathrm{Rb}$ is forbidden and only couples through collisions, we can treat the $D_{1}$ and $D_{2}$ transitions in the $\mathrm{Rb}$ atom as two separate oscillators. From the dressed state picture, a single two-level system will produce two sidebands. Therefore, one would expect $\mathrm{Rb}$, modeled as two oscillators, to produce four sidebands in total; however, this is not the case. For large pump detunings, a single sideband for each oscillator is nearly degenerate with its respective two-level system, resulting in the sideband's absorption. Subsequently, only two sidebands, the non-degenerate sideband for each oscillator, will be observed.

The $\mathrm{Rb}$ cell was optically pumped using a narrowband $(<0.2 \mathrm{~nm}$ linewidth) 6 ps laser tunable from 763 to $798 \mathrm{~nm}$ with a maximum pulse energy of $10 \mu \mathrm{J}$. This light source was produced via a home-built seeded optical parametric amplifier (OPA) utilizing a pulsed $10 \mathrm{ps}$ laser at $1064 \mathrm{~nm}$ (18 kHz repetition rate) that was frequency-doubled in a lithium triborate crystal to a $100-\mu \mathrm{J}, 6-\mathrm{ps}$ laser at $532 \mathrm{~nm}$. This 532-nm laser pulse was then used to pump a pair of lithium triborate crystals in a seeded OPA configuration, where a Littman-Metcalf extended cavity diode laser with a tuning range from 763 to $798 \mathrm{~nm}$ (Sacher Lasertechnik Lion Series, Marburg, Germany) acted as the seed. The resulting OPA output was then focused into the $\mathrm{Rb}$ cell using a $7.5-\mathrm{cm}$ planoconvex lens, producing a $50-\mu \mathrm{m}$ diameter spot inside the cell.

Prior to the focusing lens, a $\mathrm{CaF}_{2}$ window is placed into the beam path at $45^{\circ}$ to pick off the backward emission from the $\mathrm{Rb}$ vapor, where it couples to a fiber input for a spectrometer (Andor Shamrock $303 i$ with Newton camera). A power meter was positioned to collect the reflection off of the glass slide from the input pump pulse to monitor the pump energy. A second fiber input for the spectrometer is located after the cell, with a $\mathrm{CaF}_{2}$ window to collect the forward emission. The experimental scheme is sketched in Figure 1c. While the pump wavelength was scanned simply by tuning the wavelength of the extended cavity diode laser, precise control of the pump energy was achieved with the aid of a half waveplate and a Glan polarizer. The Rb itself was enclosed in a 2-mm-long heated sapphire cell and heated to temperatures between 200 and $310^{\circ} \mathrm{C}$, providing $\mathrm{Rb}$ densities ranging from $\sim 8 \times 10^{14}$ to $3 \times 10^{16} \mathrm{~cm}^{-3}$, and $\Omega_{\mathrm{A}} \sim 3 \times 10^{13} \mathrm{rad} \mathrm{s}^{-1}$.

Given these experimental parameters, the Rabi frequency, $\Omega_{\mathrm{d}}$, is estimated to be on the order of $\sim 2 \pi \times 10^{12} \mathrm{rad} \mathrm{s}^{-1}$ ( $1 \mathrm{THz}$ ), as the electric field amplitude is estimated via $E=\sqrt{2 I / n \varepsilon_{0} c}$, where $n$ is the index of refraction, $c$ is the speed of light and $I$ is the intensity of the pump laser. The electric dipole moment, $\wp$, was determined through the measured values of the spontaneous decay rate, $\Gamma_{a b}$, by $\wp=\sqrt{3 \pi \varepsilon_{0} \hbar c^{3} \Gamma_{a b} / \omega_{a b}{ }^{3}}$, where $\omega_{a b}$ is the transition frequency.
The collective atomic frequency, $\Omega_{\mathrm{A}}$, can also be estimated using $\Omega_{\mathrm{A}}=\sqrt{3 c \lambda_{a b}^{2} \Gamma_{a b} N / 8 \pi}$, where $\lambda_{a b}$ is the transition wavelength and $N$ is the atomic density. Thus, given a Rb vapor density of $N \sim 10^{17} \mathrm{~cm}^{-3}$, $\Omega_{\mathrm{A}}$ is $\sim 3 \times 10^{13} \mathrm{rad} \mathrm{s}^{-1}$. In comparison, in the dominant loss channel, the collisional dephasing rate, $\gamma_{\text {coll }}=(2 \pi N) \times 0.515 \times 10^{-13}$, is $\gamma_{\text {coll }} \sim 3 \times 10^{9} \mathrm{rad} \mathrm{s}^{-1}$. Given that $\Omega_{\mathrm{d}}$ and $\Omega_{\mathrm{A}}$ are both greater than $\gamma_{\text {coll }}$, we can safely assert that our experiment is within the regime where coherence effects have a large role.

\section{RESULTS AND DISCUSSION}

The initial results revealed two bright emission peaks with detunings from the pump frequency, $\nu_{\mathrm{d}}$, approximately matching the respective effective Rabi frequencies for the $D_{1}$ and $D_{2}$ transitions. This sideband emission was observed in both the forward (Figure 2a) and backward (Figure 3a) directions along the pump beam. Given a 5- $\mu \mathrm{J}$ pump energy, the conversion efficiency for the forward sideband emission was $\sim 1-2 \%$, thus varying by $\sim 50 \mathrm{~nJ}$. This variation in the efficiency depended on the detuning of the pump field, with higher efficiencies occurring when the Rabi frequency, $\Omega_{\mathrm{d}}$, was approximately equal to the pump detuning, $\Delta$. This finding is in agreement with an earlier work $^{30}$, where the emission was maximized for a ratio of the Rabi frequency to detuning of 1.5 to 3.0 , with the exact value depending on the experimental conditions. For a gain medium length of $1 \mathrm{~mm}$ and diameter of $\sim 50 \mu \mathrm{m}$, this gives a gain coefficient on the order of $4 \times 10^{-2} \mathrm{~m} \mathrm{GW}^{-1}$, which is higher than the stimulated Raman/ Brillouin in most gases and liquids ${ }^{31}$. The full-width at halfmaximum linewidth of the emission was $\sim 1.5 \mathrm{~nm}$ for most detunings, but it broadened with the increasing vapor pressure. Whereas emission from the side was also detected, it was approximately three orders of magnitude weaker (see Supplementary Information). For a full characterization of the emission, the pump wavelength, pump energy and $\mathrm{Rb}$ density were independently varied. The results of the wavelength and energy study for the forward direction are depicted in Figure 2.

To understand the nature of the emission, we derived analytic expressions from the density matrix equations using the conceptual model described earlier. Specifically, a two-level system with a transition frequency $\omega_{a b}$ is driven by a strong pump field with frequency $\nu_{\mathrm{d}}$ and Rabi frequency $\Omega_{\mathrm{d}}$, as well as two weak probe fields symmetrically red- and blue-detuned from the pump laser at frequencies $\omega_{3}=\nu_{\mathrm{d}}-\Omega_{\mathrm{Eff}}$ and $\omega_{4}=\nu_{\mathrm{d}}+\Omega_{\mathrm{Eff}}$. The analytic expressions provide solutions for the electric field amplitudes of the Stokes and anti-Stokes frequencies, $A_{3}$ and $A_{4}$,

$$
\begin{aligned}
& A_{3}(z)=\left[\frac{\left(e^{g_{+} z}-e^{g_{-} z}\right)\left(\kappa_{3} A_{4}^{0}-\left(g_{-}+\alpha_{3}+\frac{i \Delta k}{2}\right) A_{3}^{0}\right)}{\left(g_{+}-g_{-}\right)}\right] e^{\frac{i \Delta k}{2} z} \\
& A_{4}^{*}(z)=\left[\frac{\left(e^{g_{+} z}-e^{g_{-} z}\right)\left(\kappa_{4}^{*} A_{3}^{0}-\left(g_{-}+\alpha_{4}+\frac{i \Delta k}{2}\right) A_{4}^{0}\right)}{\left(g_{+}-g_{-}\right)}\right] e^{\frac{i \Delta k_{z}}{2} z}
\end{aligned}
$$

where $g_{ \pm}$are the gain coefficients, and $\alpha_{i}$ and $\kappa_{i}$ are the absorption and coupling coefficients, respectively. The gain coefficients, $g_{ \pm}$, which are also functions of $\alpha_{i}$ and $\kappa_{i}$, are given by:

$$
g_{ \pm}=\frac{ \pm \sqrt{\left(\alpha_{3}-\alpha_{4}+i \Delta k\right)^{2}+4 \kappa_{3} \kappa_{4}^{*}}-\left(\alpha_{3}+\alpha_{4}\right)}{2}
$$

Both $\alpha_{i}$ and $\kappa_{i}$ themselves are functions of the linear $\left(\chi^{1}\right)$ and nonlinear $\left(\chi^{3}\right)$ susceptibilities and thus are dependent on the pump frequency, $\nu_{\mathrm{d}}$, Rabi frequency, $\Omega_{\mathrm{d}}$, and density of the gas, $N$. Consequently, the gain of the probe fields is also dependent on these parameters, as well 
a

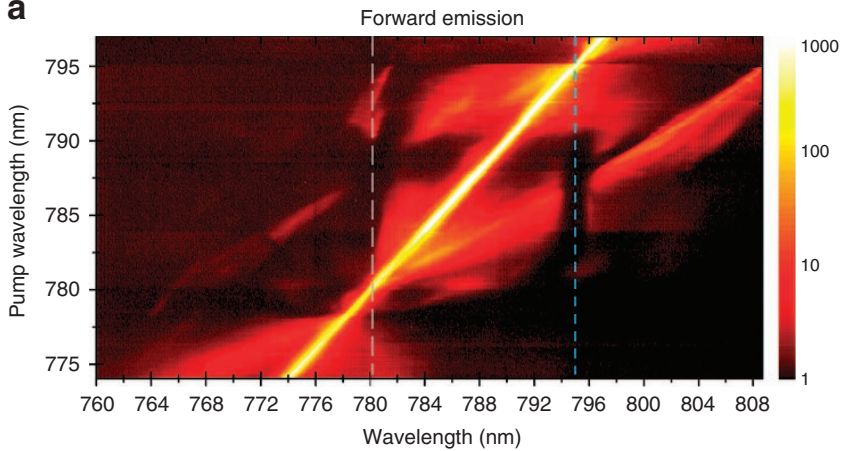

b

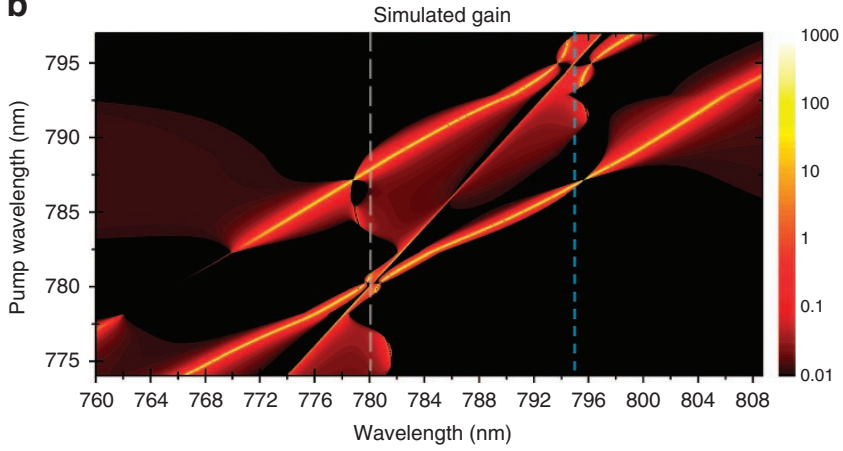

C

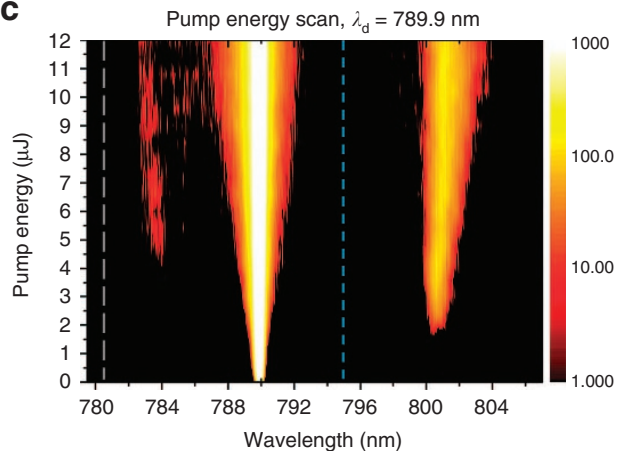

d

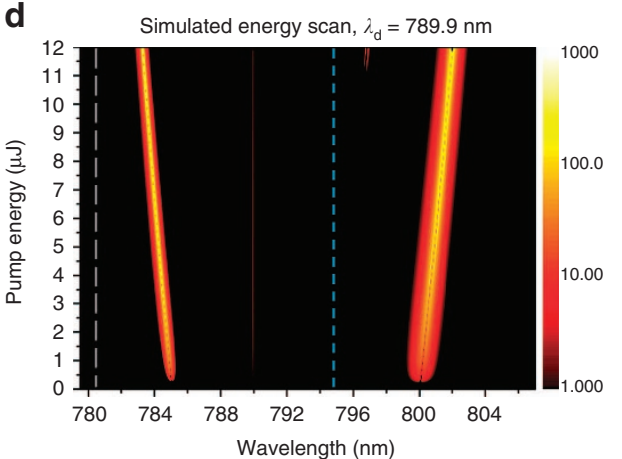

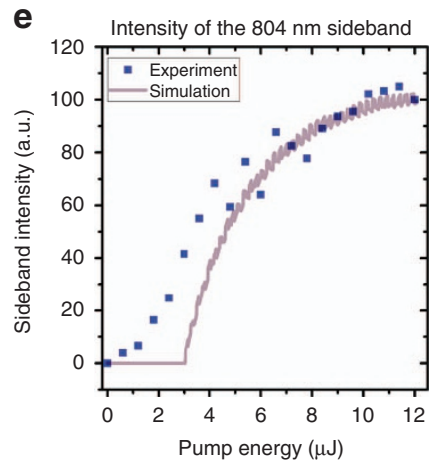

Figure 2 Forward emission and simulations. (a) Two-dimensional (2D) intensity map of the observed emission in the forward direction using a 10- $\mu$ J pump scanned from 775 to $797 \mathrm{~nm}$. (b) 2D map of the simulated gain using a Rabi frequency of $1.2 \mathrm{THz}$. The strong central bright field in both (a) and (b) is indicative of the laser field, whereas the two arms that intersect the laser field at $D_{1}$ and $D_{2}$ (795 and $780 \mathrm{~nm}$, respectively) are the emitted sidebands. The sidebands due to $D_{2}$ and $D_{1}$ range from 767 to $808 \mathrm{~nm}$ and 762 to $796 \mathrm{~nm}$, respectively. The locations of the $D_{1}$ and $D_{2}$ transitions are indicated with a short-dashed blue line and a long-dashed grey line, respectively. (c) 2D intensity map of the observed emission in the forward direction as the pulsed energy is increased, and the pump held constant at $790 \mathrm{~nm}$. (d) $2 \mathrm{D}$ map of the simulated gain in the forward direction as the pump energy is increased, and the pump wavelength remains constant at $790 \mathrm{~nm}$. (e) Sideband intensity versus pump energy. The blue dots are the experimental measurements along the sideband at $804 \mathrm{~nm}$, and the solid grey curve is the corresponding simulation result.

as the probe frequency. Detailed derivations and definitions for this approach are included in the Supplementary Information.

Following this treatment and using values closely matching the experimental parameters, simulations of the gain were performed to confirm the behavior of the emission (Figure $2 \mathrm{~b}$ and $2 \mathrm{~d}$ ). When the pump wavelength is scanned, the model predicts a gain at wavelengths detuned from the pump by the effective Rabi frequencies. For the $D_{1}$ and $D_{2}$ transitions of $\mathrm{Rb}$, this corresponds to gains at two distinct wavelengths, a single sideband for each transition, that move further from both the pump and transition wavelength as the detuning is increased (Figure 3b). This result is extremely consistent with our experimental observations (Figure 2a).

The pump energy study similarly revealed close agreement between the experiment and simulations, where both exhibited a threshold-like behavior (see Figure 2c-2e). This threshold is caused by the coherence reaching a critical level such that the gain overcomes the decoherence of the system. Furthermore, as the pump energy was increased, the observed sidebands became further detuned, which is in keeping with our model, as the effective Rabi frequency, $\Omega_{\mathrm{Eff}}$, is dependent on the pump energy. This shift was especially evident when the pump beam detuning, $\Delta$, was very small in comparison with $\Omega_{\mathrm{d}}$. This is highly indicative that the sideband emission is driven by the coherence of the system.

A threshold-like behavior was also expected and observed when the $\mathrm{Rb}$ density was increased. Unlike when scanning the pump energy or frequency, the frequencies of the sideband emission remain unchanged as the density is varied. This behavior arises from the need to achieve a critical atomic drive frequency, $\Omega_{\mathrm{A}}$, which is directly dependent upon the density (see Materials and Methods section). Once $\Omega_{\mathrm{A}}$ is greater than the decoherence rate, the gain can scale with the length as the emission propagates through. Given the long pencillike geometry of our gain region, this also provides directionality along the pump path for the emission. This threshold was observed at a Rb density of $\sim 2 \times 10^{15} \mathrm{~cm}^{-3}\left(\sim 220^{\circ} \mathrm{C}\right)$.

The observed backward emission exhibited nearly identical behavior to the forward emission (see Figure 3 and Supplementary Information). In fact, while the sideband emission behaved identically, our observations in the backward direction proved to be more information-rich due to the lack of the strong driving pump. Without the intense pump field, weaker signals such as fluorescence were observable, providing useful information regarding the population dynamics. In particular, when the pump field was near-resonant with the $D_{1}$ or $D_{2}$ transition, emission at the off-resonant transition was observed as well as fluorescence from some of the upper excited states of $\mathrm{Rb}$. These additional features are indicative of significant population transfer and more complicated transient dynamics when the pump is near resonance. Conversely, when the pump is far from resonance, these features disappear, allowing for a simpler treatment of the system as two separate two-level systems. These additional fluorescent lines were also observed using a CW field (see Supplementary Information). No sideband emission was detected using the CW pump, which again demonstrates that FRISBE arises from transient dynamics, where the quantum coherence plays an important role. 
a
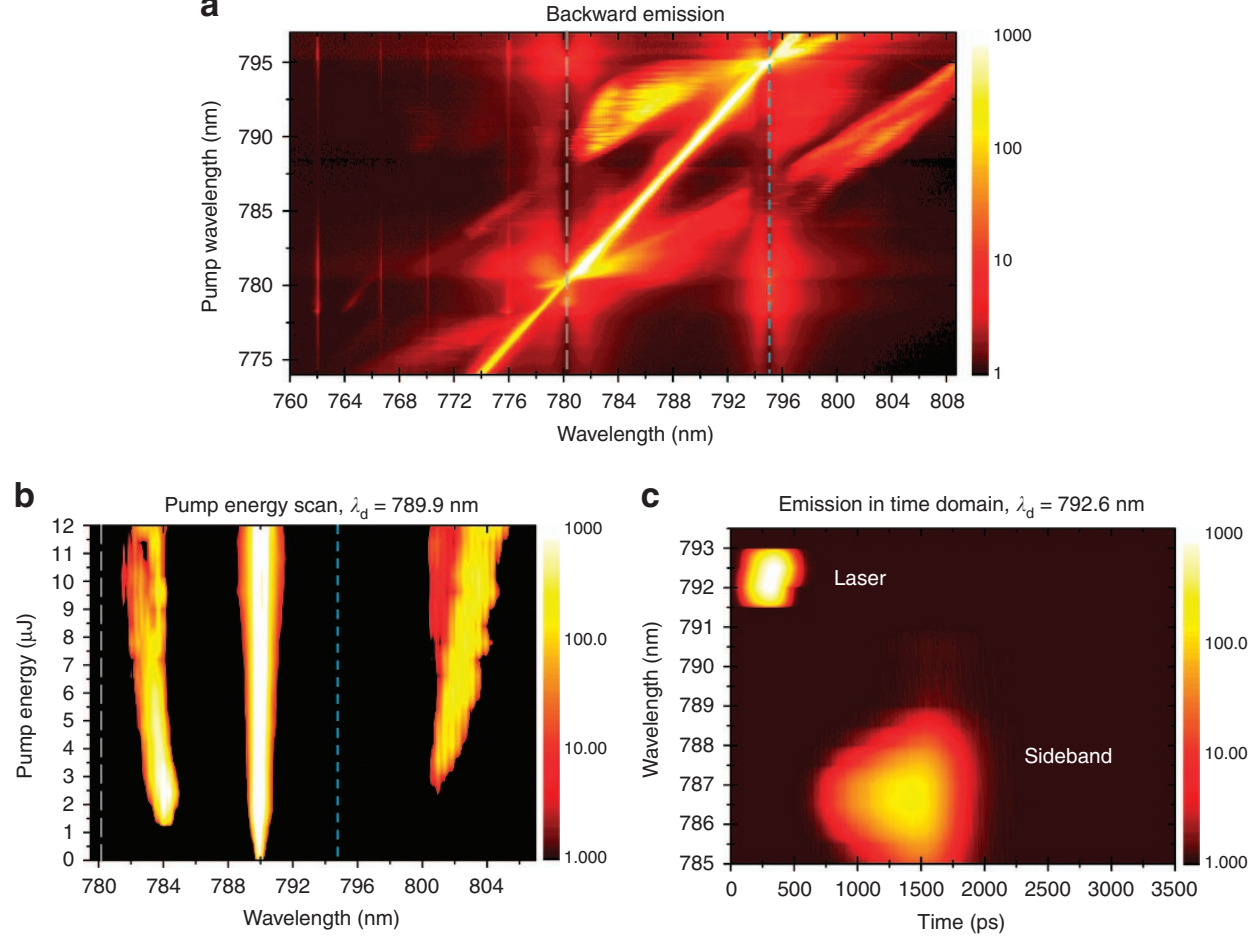

Figure 3 Backward emission characteristics and temporal dynamics. Spectral measurements of the backward emission from Rb vapor as a function of the pump wavelength (a) and pump energy (b). (c) Time-resolved emission of the sidebands. The sideband at $786 \mathrm{~nm}$ emits in less than $1 \mathrm{~ns}$, which is on the order of the estimated decoherence time of the system.

It is important to note that, although our theoretical model strongly agrees with the forward emission, it does not predict emission in the backward direction. To better understand how the backward emission is likely arising, it is best to examine the process of super-radiance, as both super-radiance and our emission are highly dependent on the collective coherence of the gain medium. In previous super-radiance experiments, it has been shown that the direction of the emission is highly dependent on the geometry of the gain medium, more so than on the directionality of the pump source ${ }^{32-34}$. In these cases, the gain mechanism itself had no directional limitations, but directional emission occurred due to the long pencil-like medium. This bears a striking resemblance to our own experiment, where the pump field is already gone by the time that the emission evolves (see Figure 3c), and an initial gain mechanism such as Mollow gain has no set directionality. Thus, four-wave mixing is more likely to occur along the extended axis of the gain medium because any initial photons traveling along that axis will interact with more of the medium and subsequently seed the process. This process would contribute to both the forward and backward emissions, although the forward emission has the added benefit of phase-matching with the pump during the pump duration.

The sideband emission was further characterized in the time domain. The backward emission was measured using time-correlated spectral measurements, that is, a monochromator (Spectral Products, CM-110) was paired with a time-correlated single photon counting a photo-multiplier tube (bh, SPC-150) that acted as the detector on the output of the monochromator. A 750-nm long-pass filter was placed in front of the photo-multiplier tube to prevent any stray 532-nm light in the OPA from corrupting our measurements. The pump laser wavelength and monochromator were then alternately scanned to create spectral time profiles. The sideband emission was observed to be time-delayed from the laser pulse by $\sim 1 \mathrm{~ns}$. This is in keeping with our predictions, as the coherence needs time to develop before any emission can occur out of the dressed state. The sideband emission duration is much faster $(<1 \mathrm{~ns})$ than the spontaneous emission rate for either of the $\mathrm{Rb}$ transitions, thus ruling out any fluorescence process, and follows the decoherence rate set by the $\mathrm{Rb}-\mathrm{Rb}$ collision rate of $\sim 1 \mathrm{GHz}$. This short, intense nature of the sideband emission makes it perfectly suited for use in other techniques such as coherent Raman and two-photon spectroscopy and other nonlinear processes.

\section{CONCLUSIONS}

One of the benefits of this method is its scalability. We explored FRISBE's ability to generate coherent XUV light by extending our simulations to other atomic species. There are two potentially viable approaches for XUV FRISBE generation. The first approach utilizes a pump field red-detuned from an XUV transition, whereby an antiStokes sideband is produced that is very nearly resonant with the XUV transition $\left(\nu_{\mathrm{d}}+\Omega_{\mathrm{Eff}} \approx \omega_{a b}\right)$. The key and challenge to this approach is generating the sideband such that it is detuned from the transition, thereby avoiding absorption and other near-resonant distortions, which requires the Rabi frequency, $\Omega_{\mathrm{d}}$, to be on the order of the pump detuning, $\Delta$.

The other method relies on a UV pump source that is blue-detuned from a transition with a large dipole moment. Here, in Figure 4, we simulated a 213-nm pump source (5th harmonic of $1064 \mathrm{~nm} \mathrm{Nd}$ : YAG) in manganese $(\mathrm{Mn})$ vapor. $\mathrm{Mn}$ is an attractive choice due to its strong ground state transition, lack of XUV ground-state transitions and high ionization level $(\sim 7.4 \mathrm{eV})$. As depicted in Figure 4, the gain produced at $\sim 172 \mathrm{~nm}$ corresponds to a sideband from the $279-\mathrm{nm}$ transition. The strength of this sideband gain is primarily dependent upon the strength of the dipole moment for the transition, although the gain does decrease as with larger pump detunings from the transition. 


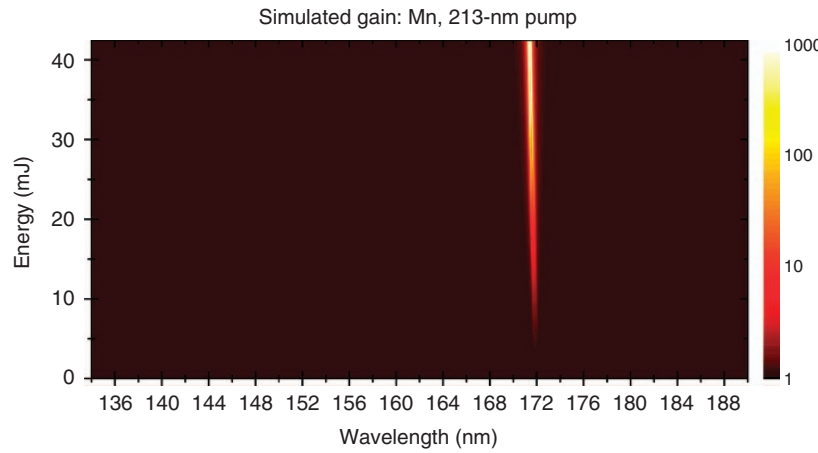

Figure 4 XUV simulation in manganese. Simulation for a 213-nm pump field in $\mathrm{Mn}$ vapor (atomic density $1 \times 10^{16} \mathrm{~cm}^{-3}$ ).

For this simulation, special care was taken in our choice of both the pump source and the atomic ensemble such that all the parameters were realistic and achievable experimentally. It should be noted that the simulation for Mn used approximately the same focal geometry as was used in our $\mathrm{Rb}$ experiment (that is, a focal depth of $1 \mathrm{~mm}$ and a spot size of $\sim 50 \mu \mathrm{m}$ ), but these parameters could be optimized for a higher emission energy as the overall gain scales with length. Similar simulations in Mn with pump wavelengths of up to $250 \mathrm{~nm}$ also depicted a FRISBE gain below $200 \mathrm{~nm}$. While these simulations are promising, there are many other potentially viable atomic species for XUV generation including silver, magnesium, zinc and calcium.

We have demonstrated the feasibility of generating coherent tunable light using only a two-level atomic system. This process should be applicable to most atomic and possibly molecular systems as well as scalable to longer gain lengths for higher energies. This could be a tremendous tool for achieving laser wavelengths in the XUV/X-ray range without the need for traditional population inversion as well as a potential source for atmospheric remote sensing. Even in other wavelength regimes where techniques such as parametric oscillators/ amplifiers offer suitable options, fast Rabi-induced sidebands could act as a supplementary technology, as they essentially do not have a damage threshold, unlike most nonlinear crystals. This would allow for the generation of higher energies per pulse and could significantly lower the cost of such technologies.

\section{CONFLICT OF INTEREST}

The authors declare no conflict of interest.

\section{ACKNOWLEDGEMENTS}

This research was in part supported by the National Science Foundation (DBI awards \#1455671 and \#1532188, and ECCS award \#1509268), by the US Department of Defense (grant \#FA9550-15-1-0517 and awards No. N00014-161-2578 and No. N00014-16-1-3054) and by Robert A. Welch Foundation (Grant No. A-1261).

1 Attwood D. Soft X-Rays and Extreme Ultraviolet Radiation: Principles and Applications. Cambridge, UK: Cambridge University Press. 2007.

2 Kocharovsky V, Cameron S, Lehmann K, Lucht R, Miles R et al. Gain-swept superradiance applied to the stand-off detection of trace impurities in the atmosphere. Proc Natl Acad Sci USA 2005; 102: 7806-7811.

3 Hemmer PR, Miles RB, Polynkin P, Siebert T, Sokolov AV et al. Standoff spectroscopy via remote generation of a backward-propagating laser beam. Proc Natl Acad Sci USA 2011; 108: 3130-3134.
4 Yuan L, Lanin AA, Jha PK, Traverso AJ, Voronine DV et al. Coherent raman umklappscattering. Laser Phys Lett 2011; 8: 736-741.

5 Gudzenko LI, Shelepin LA. Negative absorption in a nonequilibrium hydrogen plasma. JETP 1964; 18: 998-1000.

6 Oliva E, Fajardo M, Li L, Pittman M, Le TTT et al. A proposal for multi-tens of GW fully coherent femtosecond soft X-ray lasers. Nat Photonics 2012; 6: 764-767.

7 Svidzinsky AA, Yuan LQ, Scully MO. Quantum amplification by superradiant emission of radiation. Phys Rev $X$ 2013; 3: 041001

8 Irons FE, Peacock NJ. Experimental evidence for population inversion in $\mathrm{C}^{5+}$ in an expanding laser-produced plasma. J Phys B 1974; 7: 1109-1112.

9 Matthews D, Hagelstein PL, Rosen MD, Eckart MJ, Ceglio NM et al. Demonstration of a soft X-ray amplifier. Phys Rev Lett 1985; 54: 110-113.

10 Suckewer S, Skinner $\mathrm{CH}$, Milchberg $\mathrm{H}$, Keane $\mathrm{C}$, Voorhees D. Amplification of stimulated soft X-ray emission in a confined plasma column. Phys Rev Lett 1985; 55: 1753-1756.

11 Rundquist A, Durfee CG III, Chang ZH, Herne C, Backus S et al. Phase-matched generation of coherent soft X-rays. Science 1998; 280: 1412-1415.

12 Popmintchev T, Chen M-C, Popmintchev D, Arpin P, Brown S et al. Bright coherent ultrahigh harmonics in the $\mathrm{keV} x$-ray regime from mid-infrared femtosecond lasers. Science 2012; 336: 1287-1291.

13 Dogariu A, Michael JB, Scully MO, Miles RB. High-gain backward lasing in air. Science $2011 ; 331: 442-445$.

14 Traverso AJ, Sanchez-Gonzalez R, Yuan LQ, Wang K, Voronine DV et al. Coherence brightened laser source for atmospheric remote sensing. Proc Natl Acad Sci USA 2012; 109: 15185-15190.

15 Kartashov D, Ališauskas S, Andriukaitis G, Pugžlys Audrius, Shneider M et al. Freespace nitrogen gas laser driven by a femtosecond filament. Phys Rev A 2012; 86: 033831.

16 Laurain A, Scheller M, Polynkin P. Low-threshold bidirectional air lasing. Phys Rev Lett 2014; 113: 253901.

17 Horvath H. Atmospheric light absorption-a review. Atmos Environ A General Top 1993; 27: 293-317.

18 Mollow BR. Stimulated emission and absorption near resonance for driven systems. Phys Rev A 1972; 5: 2217-2222.

19 Wu FY, Ezekiel S, Ducloy M, Mollow BR. Observation of amplification in a strongly driven two-level atomic system at optical frequencies. Phys Rev Lett 1977; 38: 1077-1080.

20 Boyd RW, Raymer MG, Narum P, Harter DJ. Four-wave parametric interactions in a strongly driven two-level system. Phys Rev A 1981; 24: 411-423.

21 Agarwal GS, Boyd RW. Quantum theory of Rabi sideband generation by forward fourwave mixing. Phys Rev A 1988; 38: 4019-4027.

22 LeBerre-Rousseau M, Ressayre E, Tallet A. Self-induced generation of an off-axis frequency shifted radiation from atoms. Opt Commun 1981; 36: 31-34.

23 Chauchard EA, Meyer YH. On the origin of the so called conical emission in laser pulse propagation in atomic vapor. Opt Commun 1984; 52: 141-144.

24 Golub I, Erez G, Shuker R. Cherenkov emission due to laser-induced moving polarisation in sodium. J Phys B 1986; 19: L115-L120.

25 Crenshaw ME, Cantrell CD. Conical emission as a result of pulse breakup into solitary waves. Phys Rev A 1989; 39: 126-148.

26 Grischkowsky D. Self-focusing of light by potassium vapor. Phys Rev Lett 1970; 24: 866-869.

27 Harter DJ, Narum P, Raymer MG, Boyd RW. Four-wave parametric amplification of Rabi sidebands in sodium. Phys Rev Lett 1981; 46: 1192-1195.

28 Maki JJ, Malcuit MS, Raymer MG, Boyd RW, Drummond PD. Influence of collisional dephasing processes on superfluorescence. Phys Rev A 1981; 40: 5135-5142.

29 Yuan LQ, Hokr BH, Traverso AJ, Voronine DV, Rostovtsev Y et al. Theoretical analysis of the coherence-brightened laser in air. Phys Rev A 2013; 87: 023826.

30 Harter DJ, Boyd RW. Four-wave mixing resonantly enhanced by ac-Stark-split levels in self-trapped filaments of light. Phys Rev A 1984; 29: 739-748.

31 Boyd RW. Nonlinear Optics, 3rd edn. Burlington, MA, USA: Academic Press. 2008.

32 Yoshikawa Y, Torii Y, Kuga T. Superradiant light scattering from thermal atomic vapors. Phys Rev Lett 2005; 94: 083602.

33 Greenberg JA, Gauthier DJSuperradiance in an ultracold thermal vaporIn. Advances in Optical Sciences Congress 2009. Honolulu, Hawaii, USA: Optical Society of America. 2009.

34 Inouye S, Chikkatur AP, Stamper-Kurn DM, Stenger J, Pritchard DE et al. Superradiant rayleigh scattering from a bose-einstein condensate. Science 1999; 285: 571-574.

This work is licensed under a Creative Commons Attribution(c) NC SA NonCommercial-ShareAlike 4.0 International License. The images or
other third party material in this article are included in the article's Creative Commons license, unless indicated otherwise in the credit line; if the material is not included under the Creative Commons license, users will need to obtain permission from the license holder to reproduce the material. To view a copy of this license, visit http://

creativecommons.org/licenses/by-nc-sa/4.0/

(C) The Author(s) 2017

Supplementary Information for this article can be found on the Light: Science \& Applications' website (http://www.nature.com/lsa). 